\title{
Basic symptoms in offspring of parents with mood and psychotic disorders
}

Alyson Zwicker, Lynn E. MacKenzie, Vladislav Drobinin, Emily Howes Vallis, Victoria C. Patterson, Meg Stephens, Jill Cumby, Lukas Propper, Sabina Abidi, Alexa Bagnell, Frauke Schultze-Lutter, Barbara Pavlova, Martin Alda and Rudolf Uher

\section{Background}

Basic symptoms, defined as subjectively perceived disturbances in thought, perception and other essential mental processes, have been established as a predictor of psychotic disorders. However, the relationship between basic symptoms and family history of a transdiagnostic range of severe mental illness, including major depressive disorder, bipolar disorder and schizophrenia, has not been examined.

\section{Aims}

We sought to test whether non-severe mood disorders and severe mood and psychotic disorders in parents is associated with increased basic symptoms in their biological offspring.

\section{Method}

We measured basic symptoms using the Schizophrenia Proneness Instrument - Child and Youth Version in 332 youth aged 8-26 years, including 93 offspring of control parents, 92 offspring of a parent with non-severe mood disorders, and 147 offspring of a parent with severe mood and psychotic disorders. We tested the relationships between parent mental illness and offspring basic symptoms in mixed-effects linear regression models.

\section{Results}

Offspring of a parent with severe mood and psychotic disorders $(B=0.69,95 \% \mathrm{Cl} 0.22-1.16, P=0.004)$ or illness with psychotic features $(B=0.68,95 \% \mathrm{Cl} 0.09-1.27, P=0.023)$ had significantly higher basic symptom scores than control offspring. Offspring of a parent with non-severe mood disorders reported intermediate levels of basic symptoms, that did not significantly differ from control offspring.

\section{Conclusions}

Basic symptoms during childhood are a marker of familial risk of psychopathology that is related to severity and is not specific to psychotic illness.

\section{Declaration of interest \\ None.}

\section{Keywords}

Major depressive disorder; bipolar disorder; schizophrenia; developmental psychopathology; basic symptoms.

\section{Copyright and usage}

(C) The Royal College of Psychiatrists 2019. This is an Open Access article, distributed under the terms of the Creative Commons Attribution-NonCommercial-NoDerivatives licence (http://creativecommons.org/licenses/by-nc-nd/4.0/), which permits noncommercial re-use, distribution, and reproduction in any medium, provided the original work is unaltered and is properly cited. The written permission of Cambridge University Press must be obtained for commercial re-use or in order to create a derivative work.
Severe mood and psychotic disorders (SMPD) are mental disorders that cause functional impairment and substantially interfere with one or more major life activities. These disorders often follow a chronic or recurrent course and available treatments have limited efficacy. ${ }^{1-4}$ Improving upon our ability to predict SMPD may be useful to inform targeted early interventions to prevent its onset. ${ }^{5}$ Recent research has shown that there is substantial overlap in the genetic and environmental contributors to various forms of SMPD. ${ }^{6-10}$ Consequently, it may be useful to identify overlapping, transdiagnostic predictors of mood and psychotic disorders that can be detected early enough to allow for preventive interventions. Self-experienced disruptions in thought, perception and other essential mental processes, referred to as basic symptoms, are potential early indicators of SMPD risk. ${ }^{11}$

\section{Basic symptoms and risk of mental illness}

Basic symptoms may represent an early manifestation of mood and psychotic disorders, particularly psychotic illness. ${ }^{5,11,12}$ Positive symptoms of psychosis include hallucinations and/or delusions, which are perceived by the affected individual as real experiences. In contrast to positive psychotic symptoms, basic symptoms are immediately recognised by the individual as abnormal disturbances to their typical thoughts, senses and feelings. ${ }^{13}$ These symptoms are often present years before the onset of illness and can be assessed in children as young as 8 years old. ${ }^{14}$ Basic symptoms have been examined in detail as a potential precursor to psychotic illness. Basic symptoms strongly predict the onset of psychotic illness. ${ }^{5}$ Basic symptoms have also been linked to other forms of mental illness, including affective disorders ${ }^{15,16}$ and are associated with lower global functioning among individuals with a range psychiatric disorders. ${ }^{17}$ However, the utility of basic symptoms as an indicator of risk for a broader range mental disorders remains to be examined.

\section{Family high-risk approach}

The best-known predictor of mood and psychotic disorders is a family history of illness. ${ }^{18}$ Risk of illness is proportional to the degree of biological relatedness to the affected individual. ${ }^{18}$ However, familial risk of mental illness is not disorder-specific. Individuals with a family history of schizophrenia are also at risk of mood disorders, and vice versa. ${ }^{19}$ This finding is supported by molecular data that show that a substantial proportion of genetic variants and gene expression abnormalities associated with mental illness are shared across psychiatric disorders. ${ }^{8,9,20,21}$ Taken together, these findings suggest that it may be useful to identify measurable experiences and behaviours that predict SMPD and are shared across disorders. By examining early manifestations of risk among offspring of parents with SMPD, we are able to distinguish possible causes or predictors of illness from the effects of SMPD and its treatment. Individuals who have a first-degree biological relative living with schizophrenia experience more basic 
symptoms than controls. ${ }^{22,23}$ However, basic symptoms have not yet been examined among youth at high familial risk for other forms of mental illness.

\section{Aims}

Here we examine the relationship between basic symptoms and family history of a spectrum of non-severe mood disorders (NSMD) and SMPD. We assessed basic symptoms in a sample of youth enriched for offspring of parents with major depressive disorder, bipolar disorder and schizophrenia, including both NSMD and SMPD. We aimed to test whether offspring basic symptoms are associated with parent mental illness, its severity, psychotic features or specific psychiatric diagnosis.

\section{Method}

\section{Participants}

The present study includes information from 909 assessments of 332 participants aged 8-26 years from 201 families, enrolled in the Families Overcoming Risks and Building Opportunities for Well-being (FORBOW) study. ${ }^{24}$ Assessors masked to information on parents, assessed basic symptoms annually with the baseline assessment occurring at an average age of 11.84 years (range 8-24 years). Each participant completed a median of three assessments (range 1-6) at 12-month intervals. Repeated basic symptom measures from all assessments were included in analyses. We included offspring of parents with major depressive disorder, bipolar disorder, psychosis spectrum disorders and offspring of control parents. Offspring of parents with SMPD were recruited through their parents' contact with mental health services in Nova Scotia, Canada. Offspring were included regardless of whether or not they had psychopathology. Age matched offspring of control parents were recruited through local school boards. To ensure that control offspring were approximately matched with offspring of affected parents on socioeconomic status, we selectively recruited control offspring from the same schools and neighbourhoods of the offspring of affected parents. We excluded offspring with a lifetime diagnosis of schizophrenia ( $n=2$ observations), schizoaffective disorder ( $n=2$ observations) or bipolar disorder ( $n=8$ observations).

We assert that all procedures contributing to this work comply with the ethical standards of the relevant national and institutional committees on human experimentation and with the Helsinki Declaration of 1975, as revised in 2008. The study protocol was approved by the Research Ethics Board of the Nova Scotia Health Authority (file number 100 266). We obtained written informed consent from participants who had the capacity to provide it. For participants who did not have the capacity to make an informed decision, a parent or guardian provided written informed consent and the participant provided assent.

\section{Parent assessment}

Diagnoses of mental disorders and psychotic symptoms according to the DSM-IV and DSM-5 were established using the Schedule for Affective Disorders and Schizophrenia (SADS-IV) ${ }^{25}$ or the Structured Clinical Interview for DSM-5 Disorders (SCID-5). ${ }^{26}$ Diagnoses were confirmed in consensus meetings with a psychiatrist masked to offspring psychopathology.

We defined SMPD as a diagnosis of major depressive disorder, bipolar disorder or a psychosis spectrum disorder accompanied by two or more of the following five severity criteria: (a) recurrent, (b) chronic, (c) presence of psychotic symptoms, (d) life-threatening suicide attempt(s) or (d) required hospital admission. ${ }^{24}$ We defined NSMD as a diagnosis of any Axis I mood disorder that did not meet two or more severity criteria. In situations where one biological parent had NSMD and one biological parent had SMPD, the offspring were placed in the SMPD group.

\section{Offspring assessment}

\section{General psychopathology}

Offspring were assessed for all Axis I disorders at 12-month intervals using the Kiddie Schedule for Affective Disorders and Schizophrenia - Present and Lifetime Version (K-SADS-PL; in offspring younger than 18 years) ${ }^{27}$ or the SCID-5 (in offspring $\geq 18$ years old). ${ }^{26} \mathrm{~A}$ single assessor completed both the diagnostic interview and the basic symptoms interview. Offspring assessors were masked to parent psychopathology. Diagnoses were confirmed in consensus meetings with a psychiatrist masked to parent diagnoses.

\section{Basic symptoms}

We assessed basic symptoms using the Schizophrenia Proneness Instrument - Child and Youth Version (SPI-CY). ${ }^{14}$ The SPI-CY was designed to be administered to children and youth and it has been used among children aged 8 years and older with good interrater reliability. ${ }^{14,28}$ The SPI-CY contains two psychosis-risk basic symptom profiles: Cognitive-Perceptive (COPER) and Cognitive Disturbances (COGDIS). COGDIS items have been shown to strongly predict psychotic illness and are part of the clinical highrisk criteria that have been recommended for the early detection of psychosis. ${ }^{5}$ Descriptions of the items in both high-risk profiles are provided in the Appendix. We calculated the SPI-CY risk score as the total number of COPER or COGDIS items scored 3 (several times in a month or weekly) to 6 (daily), divided by the total number of items with a valid frequency rating. We calculated a COGDIS score, which incorporates the nine items included in the COGDIS criteria, using the same process. For analyses, we standardised the SPI-CY risk score and COGDIS score by the means and standard deviations of the control offspring scores.

\section{Antecedent basic symptoms}

It may be desirable to use a dichotomous indicator of basic symptoms in applications that require yes or no decisions. Therefore, we defined antecedent basic symptoms as the presence of COPER and/or COGDIS criteria. ${ }^{24}$ We report the rates of offspring who met our predefined antecedent basic symptoms threshold at their first assessment and at any assessment.

\section{Statistical analysis}

We tested the effect of parent mental illness on offspring basic symptoms in mixed-effects linear regression models using the lme4 package, ${ }^{29}$ implemented in R Studio ( $\mathrm{R}$ version 3.4 .3 ) ${ }^{30} \mathrm{We}$ accounted for the non-independence of observations from related individuals and from repeated measures within the same individual by including family and individual identifiers as random effects in the models. We included fixed effects of age, biological gender and time in the study as covariates. To test the effect of parent's primary illness severity (control, NSMD, SMPD) on offspring basic symptoms, we fitted a linear mixed regression model with standardised offspring SPI-CY risk score as the dependent variable and parent illness severity as the independent variable. We tested the effect of parent psychosis (control, non-psychotic illness, psychotic illness) on offspring basic symptoms by fitting a linear mixed regression model with standardised offspring SPI-CY risk score as the dependent variable and parent psychosis as the independent variable. We tested the effect of parent's primary diagnosis (control, major depressive disorder, bipolar disorder, psychosis spectrum disorder) on offspring basic symptoms by fitting a 
linear mixed regression model with standardised offspring SPI-CY risk score as the dependent variable and parent diagnosis as the independent variable (see supplementary Tables 9 and 10 and supplementary Fig. 2 available at https://doi.org/10.1192/bjo.2019.40). We also tested the effects of parent illness severity, parent psychotic symptoms and parent diagnosis on offspring COGDIS scores separately, using the same methodology as described above. Effect sizes are summarised with standardised beta coefficients and their $95 \%$ confidence intervals.

\section{Sensitivity analyses}

We opted not to exclude offspring with major depressive disorder from the primary analyses because depression was common and excluding it would reduce the representativeness of the sample. However, to ensure that our results were not unduly influenced by offspring depressive disorders, we performed sensitivity analysis by excluding observations in which offspring had experienced a major depressive episode within the 12 months prior to the assessment. Additionally, the prevalence and clinical significance of basic symptoms has been shown to vary with age. ${ }^{31}$ As we included participants across a broad range of ages, we stratified analyses by age and tested the effect of parent illness severity (control, NSMD, SMPD) on offspring basic symptoms among participants aged 11 years and under and 12 years and older separately (see supplementary materials).

\section{Results}

\section{Participant characteristics and basic symptom scores}

The sample included 93 offspring of control parents, 92 offspring of a parent with NSMD and 147 offspring of a parent with SMPD. The characteristics of the participants and the rates of antecedent basic symptoms across parent groups are shown in Table 1.

\section{Differences in SPI-CY risk scores by parent illness severity}

Across the 909 assessments of 332 children and youth with valid SPI-CY risk scores, basic symptoms were significantly elevated among the offspring of parents with SMPD compared with controls ( $B=0.69,95 \%$ CI 0.22-1.16, $P=0.004$; see Figures 1 and 2). Basic symptom scores were numerically elevated among offspring of parents with NSMD, but this difference was not statistically significant $(B=0.22,95 \% \mathrm{CI}-0.30$ to $0.73, P=0.415)$. When we excluded observations at which offspring experienced a major depressive

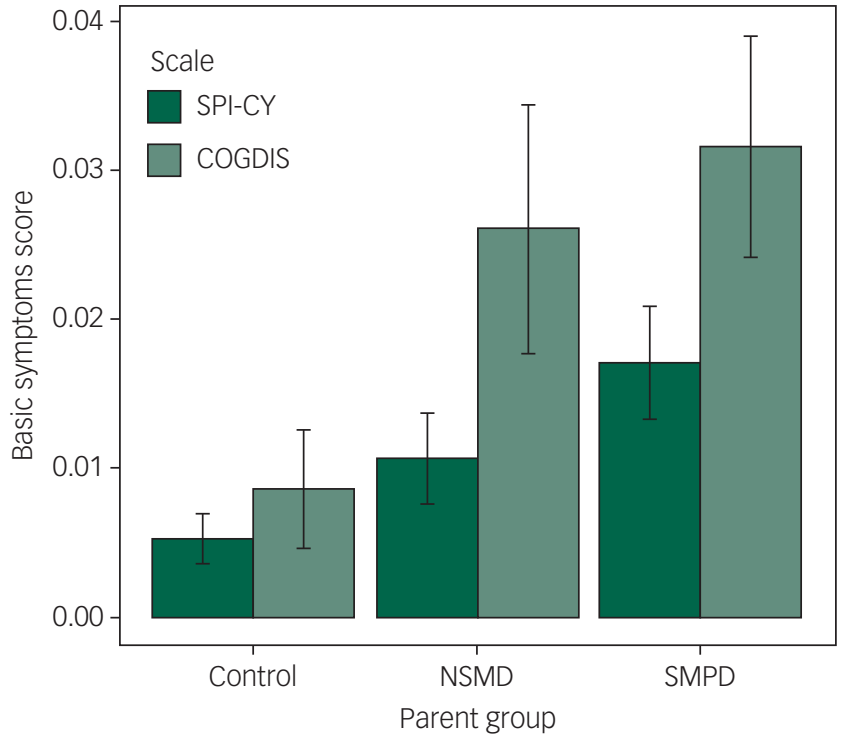

Fig. 1 Mean Schizophrenia Proneness Instrument - Child and Youth Version (SPI-CY) risk score and Cognitive Disturbances (COGDIS) score, stratified by parent illness severity group.

NSMD, non-severe mood disorder, SMPD, severe mood and psychotic disorders. Error bars represent standard error of the mean.

episode within 12 months prior to the assessment, basic symptoms remained significantly elevated among the offspring of parents with SMPD $(B=0.49,95 \%$ CI $0.10-0.87, P=0.014)$. Full regression results are shown in supplementary Tables 1 and 2. In age-stratified analyses, these findings remained consistent in both the younger (8-11 year olds) and older (12 years and older) subsets (see supplementary Tables 11 and 13 and supplementary Figs. 3 and 4).

\section{Differences in COGDIS score by parent illness severity}

Across the 905 assessments of 331 children and youth with valid COGDIS scores, basic symptoms were significantly elevated among the offspring of parents with SMPD compared with controls ( $B=0.53,95 \%$ CI 0.13-0.93, $P=0.009$; see Figs. 1 and 2 ). When we excluded observations at which offspring experienced a major depressive episode within 12 months prior to the assessment, basic symptoms remained significantly elevated among the offspring of parents with SMPD $(B=0.39,95 \%$ CI $0.04-0.73$,

\begin{tabular}{|c|c|c|c|}
\hline & \multicolumn{3}{|c|}{ Parent group } \\
\hline & Control $(n=93)$ & Non-severe mood disorder $(n=92)$ & Severe mood and psychotic disorders $(n=147)$ \\
\hline Families, $n$ & 60 & 59 & 88 \\
\hline \multicolumn{4}{|l|}{ Parent diagnosis } \\
\hline Major depressive disorder, $n$ & 0 & 85 & 62 \\
\hline Bipolar disorder, $n$ & 0 & 7 & 68 \\
\hline Schizophrenia, $n$ & 0 & 0 & 17 \\
\hline Parent illness psychotic features, $n$ & 0 & 1 & 58 \\
\hline \multicolumn{4}{|l|}{ Offspring } \\
\hline Age, mean (s.d.) ${ }^{b}$ & $12.11(3.1)$ & $13.65(4.2)$ & $13.78(4.0)$ \\
\hline Number of follow-ups, mean (s.d. $)^{b}$ & $2.43(1.3)$ & $2.66(1.4)$ & $3.02(1.6)$ \\
\hline Females, $n(\%)^{b}$ & $42(45.2)$ & $44(47.8)$ & $83(56.5)$ \\
\hline Antecedent BS at baseline, $n(\%)$ & $12(12.90)$ & $16(17.39)$ & $35(23.81)$ \\
\hline Antecedent BS ever, $n(\%)^{\mathrm{b}}$ & $18(19.35)$ & $26(28.26)$ & $54(36.73)$ \\
\hline
\end{tabular}




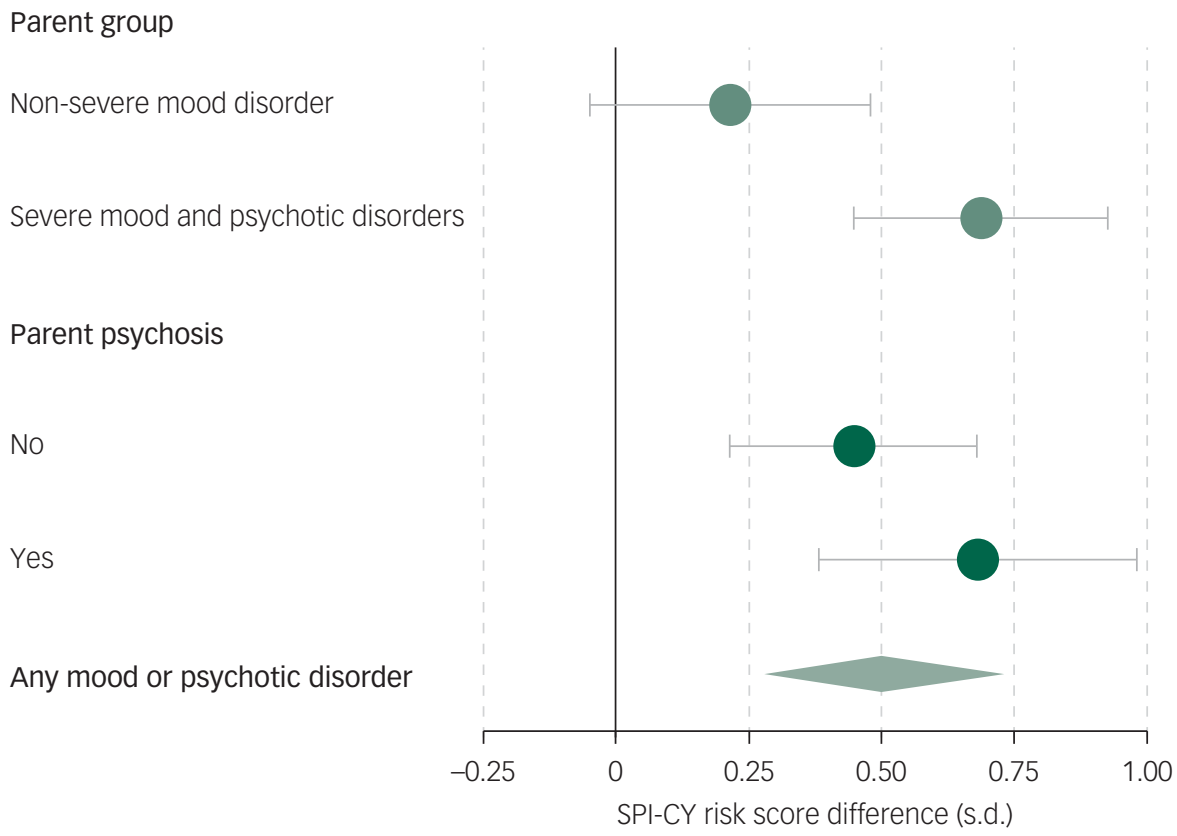

Fig. 2 Effect of parent illness on offspring basic symptoms.

Circles represent the effect size of the standard deviation (s.d.) increase in Schizophrenia Proneness Instrument - Child and Youth Version (SPI-CY) risk score compared with controls for each parent group. Whiskers represent the standard error.

$P=0.028$ ). Full regression results are shown in supplementary Tables 3 and 4 . In age-stratified analyses, COGDIS scores were numerically increased among offspring of parents with SMPD, however these differences were only statistically significant in the younger (8-11 year olds) subset (see supplementary Tables 12 and 14 and supplementary Figs. 3 and 4).

\section{Differences in basic symptom scores by parent psychosis}

Across the 909 assessments of 332 children and youth with valid SPI-CY risk scores, basic symptoms were significantly elevated among offspring of a parent with psychotic mental illness compared with controls $(B=0.68,95 \%$ CI $0.09-1.27, P=0.023$; see supplementary Fig. 1). Offspring of a parent with non-psychotic mental illness had numerically higher SPI-CY risk scores than controls, but this difference was not statistically significant $(B=0.45,95 \%$ CI -0.01 to $0.90, P=0.055$, see supplementary Fig. 1$)$. When we excluded observations in which offspring experienced a major depressive episode within 12 months prior to the assessment, both the offspring of parents with psychotic mental illness $(B=0.44$, $95 \% \mathrm{CI}-0.05$ to $0.92, P=0.078)$ and with non-psychotic mental illness $(B=0.35,95 \% \mathrm{CI}-0.02$ to $0.72, P=0.067)$ had numerically higher SPI-CY risk scores than controls, but the difference was not statistically significant. Similarly, COGDIS scores were significantly elevated among the offspring of parents with psychotic mental illness ( $B=0.55,95 \%$ CI 0.05 to $1.04, P=0.030)$ and with non-psychotic mental illness $(B=0.41,95 \% \mathrm{CI}-0.02$ to $0.80, P=0.037)$. Full regression results are shown in supplementary Tables $5-8$.

\section{Antecedent basic symptoms}

We defined antecedent basic symptoms as the presence of COPER and/or COGDIS high-risk criteria. The rate of youth meeting these high-risk criteria increased with increasing parent severity: $12.9 \%$ control offspring, $17.4 \%$ of offspring of parents with NSMD and
$23.8 \%$ of offspring of parents with SMPD had antecedent basic symptoms at baseline (Table 1).

\section{Discussion}

\section{Main findings}

We sought to test whether basic symptoms during childhood and adolescence are elevated among offspring of parents with a spectrum of NSMD and SMPD. We found that basic symptoms were most elevated among offspring of parents with SMPD, intermediate in offspring of parents with NSMD, and lowest in offspring of control parents.

\section{Comparison with findings from other studies}

Our study was motivated by a need to identify early transdiagnostic indicators of risk for SMPD among youth. Previous studies have established that basic symptoms predict psychosis, and can be present years before its onset. ${ }^{5,32}$ However, basic symptoms have not been previously examined among offspring of parents with a broad range of major mood and psychotic disorders. Consistent with prior studies which show offspring of parents with mental illness are at risk of developing psychopathology themselves, ${ }^{19}$ we found that basic symptoms are elevated among the offspring of parents with SMPD compared with controls. We also confirmed that basic symptom scores are elevated among first-degree relatives of individuals with psychosis. ${ }^{23}$ Our results are consistent with prior findings showing that offspring of parents with SMPD are at increased risk for multiple forms of psychopathology, in addition to the disorder present in the parent. ${ }^{19}$ Additionally, it has been suggested that psychosis may represent a transdiagnostic indicator of illness severity. ${ }^{33,34}$ This is supported by studies showing that the presence of psychotic symptoms in non-psychotic disorders has been associated with more severe illness and worse treatment outcomes. ${ }^{35-38}$ Our results suggest that basic symptoms represent a 
transdiagnostic marker of risk for SMPD that is not specific to psychotic illness.

\section{Strengths and limitations}

The present study benefits from the inclusion of offspring of parents with mental illness, resulting in a concentration of familial risk of psychopathology. As a result, our sample has a higher rate of basic symptoms than in the general population. We also benefit from a longitudinal design, with repeated assessments allowing the capture of basic symptoms over the period of several years. However, our results should be interpreted in the context of our study limitations. The main limitation is the smaller number of offspring of parents with a schizophrenia spectrum disorder. The majority of parents in our sample who experience psychosis have bipolar disorder or major depressive disorder with psychotic features. The smaller enrolment of offspring of parents with schizophrenia may be in part be because individuals with schizophrenia tend to have fewer children. ${ }^{39}$ However, enrolment in our cohort is ongoing and will include more offspring of parents with schizophrenia spectrum disorders in the future. Additionally, since basic symptoms are more prevalent and may be more clinically relevant among older adolescents, ${ }^{28,31}$ our study was limited by the inclusion of younger adolescents and children. However, in our age-stratified sensitivity analyses, we found that basic symptom scores were independently associated with parent mental illness among 8- to 11-year-old and 12- to 27-year-old offspring.

\section{Implications}

The results of our study have potential implications for future research. The finding that basic symptoms are elevated among young offspring of parents with SMPD can help target interventions to youth at high risk of mood and psychotic disorders, long before the onset of illness. Interventions aimed at preventing psychosis among individuals experiencing prodromal symptoms have been criticised, in part because 'good' outcomes may be synonymous with onsets of other, non-psychotic illnesses among intervention recipients. ${ }^{34}$ It has been shown that earlier interventions produce better outcomes. ${ }^{40,41}$ Our results suggest that basic symptoms may represent a useful transdiagnostic risk indicator. Basic symptoms could be used, in combination with other factors, to identify high-risk youth who may benefit from targeted interventions before the onset of major mental illnesses. Our results warrant further investigation in other familial high-risk cohorts. Additionally, the basic symptom assessment tool ${ }^{14}$ could be adopted by cohorts currently using interview measures of psychopathology.
In conclusion, we found that basic symptoms are elevated among offspring of parents with SMPD, in addition to offspring of parents with psychosis. Our results suggest that basic symptoms during childhood are a marker of familial risk for psychopathology that is related to severity and is not specific to psychotic illness. Future studies could explore the value of basic symptoms as a transdiagnostic predictor of mental illness.

Alyson Zwicker, BSC, PhD Candidate, Nova Scotia Health Authority; and Department of Pathology, Dalhousie University, Canada; Lynn E. MacKenzie, BA, MA, PhD Candidate, Nova Scotia Health Authority; and Department of Psychology and Neuroscience, Dalhousie University, Canada; Vladislav Drobinin, BSc, PhD Candidate, Nova Scotia Health Authority; and Department of Medical Neuroscience, Dalhousie University, Canada; Emily Howes Vallis, BA, MSc Candidate, Nova Scotia Health Authority; and Department of Psychiatry, Dalhousie University, Canada; Victoria C. Patterson, BA, PhD Candidate, Nova Scotia Health Authority; and Department of Psychology and Neuroscience, Dalhousie University, Canada; Meg Stephens, BMBS, BMedSci, Research Assistant, Nova Scotia Health Authority, Canada; Jill Cumby, MN, registered nurse (RN), Nova Scotia Health Authority, Canada; Lukas Propper, MD, Psychiatrist, Department of Psychiatry, Dalhousie University; and IWK Health Centre, Canada; Sabina Abidi, BSC, BA, MD, Psychiatrist, Department of Psychiatry, Dalhousie University; and IWK Health Centre, Canada; Alexa Bagnell, BSC, MA, MD, Psychiatrist, Department of Psychiatry, Dalhousie University; and IWK Health Centre, Canada; Frauke Schultze-Lutter, PhD, Assistant Professor, Department of Psychiatry and Psychotherapy, Medical Faculty, Heinrich-Heine University, Germany; Barbara Pavlova, PhD, DClinPsy, RPsych, Psychologist, Nova Scotia Health Authority; and Department of Psychiatry, Dalhousie University, Canada; Martin Alda, MD, FRCPC, Psychiatrist, Nova Scotia Health Authority; and Department of Psychiatry, Dalhousie University, Canada; Rudolf Uher (D), MD, PhD, MRCPsych,

Psychiatrist, Nova Scotia Health Authority; Department of Pathology; Department of Psychology and Neuroscience; Department of Medical Neuroscience; Department of Psychiatry, Dalhousie University; IWK Health Centre, Canada; and Institute of Psychiatry, Psychology and Neuroscience, King's College London, UK

Correspondence: Rudolf Uher, Dalhousie University, 5909 Veterans Memorial Lane, Halifax, Nova Scotia, B3H 2E2, Canada. Email: uher@dal.ca.

First received 28 Feb 2019, final revision 3 May 2019, accepted 12 May 2019

\section{Funding}

The work leading to this publication has been supported by funding from the Canada Research Chairs Program (award number 231397), the Canadian Institutes of Health Research (Grant reference numbers 124976, 142738 and 148394), the Brain \& Behavior Research Foundation (NARSAD) Independent Investigator Grant 24684, Nova Scotia Health Research Foundation (grants 275319, 1716 and 353892) and the Dalhousie Medical Research Foundation. A.Z. has been supported by the Lindsay Family Graduate Studentship. V.D. was supported by the CIHR Doctoral Award (157975)

\section{Acknowledgements}

The authors would like to thank the families who have participated in FORBOW. The authors would also like to acknowledge the contributions of the FORBOW research team (see http:// unw.forbow.org)

\section{Supplementary material}

Supplementary material is available online at https://doi.org/10.1192/bjo.2019.40.

\section{Appendix}

\section{Appendix Items included in the basic symptoms high-risk profiles.}

\section{Item name (item number)}

Decreased ability to discriminate between ideas and perception, fantasy and true memories (B1) Unstable ideas of reference (B2)

Visual perception disturbances (B3, 01, 03)

Acoustic perception disturbances (B4.2, B5)

\section{Description}

Difficulty locating the source of a memory resulting in an inability to distinguish between fantasy and true memories.

Experiences of self-reference that are almost immediately rectified upon further consideration.

Aspects of vision are misperceived but the individual is aware of their true appearance.

Non-verbal auditory pseudo-hallucinations, changes in the quality of sounds, or abnormally long-lasting residual sounds

\section{Example prompt}

Do you become confused about whether you actually did something in the past or whether you just imagined it?

Do you ever think that the actions or comments of others are about you - but yet you are certain they are not?

Do the outlines of objects sometimes appear broken, curved or wavy?

Do you sometimes have sudden short-lived difficulty with your hearing - like sounds being muffled or less loud? 
Appendix (Continued)

Item name (item number)

Derealisation (B7)

Thought interference (D9)

Thought pressure (D10)

Disturbance of receptive speech (D11) Disturbance in the understanding of words that are either

Thought perseveration (D14)

Thought blockages (D15)

Disturbances of abstract thinking (D7)

Inability to divide attention (D8)

Disturbances of expressive speech (D12)

Captivation of attention by details of the visual field $(\mathrm{O} 2)$ individual being able to suppress or guide them. read or heard.

The annoying rehearsal of unimportant, emotionally neutral thoughts related to trivial events of the recent past.

A sudden interruption in the flow of thoughts, of the mind suddenly going blank, or the fading of thoughts.

Deficits in the ability to understand abstract, figurative or symbolic phrases beyond their literal meaning.

A change in how one relates emotionally to the environment: (1) the environment appears unreal or environment. the train of thought.

fficulty in dealing with demands that involve more than one sensory modality at a time.

Subjective difficulty in finding the right words when trying to express oneself.

An ordinary visual stimulus stands out in a striking manner so that it appears almost isolated from the rest of the environment.

\section{Example promp}

Do you sometimes experience your surroundings as changed or strange? As if the world around you is not real?

If you want to concentrate on something, is your concentration suddenly interrupted by unimportant, irrelevant thoughts?

Do you sometimes have the feeling that you are not able to control your thoughts anymore?

Do you sometimes have difficulty understanding conversations that you know you should be able to follow?

Do you sometimes find yourself thinking about past events that have no special meaning, even though you want to think about something else or go to sleep?

Do your thoughts sometimes disappear suddenly, as if they were cut short?

Do you have difficulty understanding the meaning of metaphors or abstract things like a saying or an idiom?

Can you do two things at once as easily as you could before?

When you want to say something, do you struggle to find the right words?

Is your attention sometimes caught by a detail in your surroundings, so that you need to look at it without wanting to?

The first five items are only in the Cognitive-Perceptive (COPER) high-risk profile, the next five items are included in both the COPER and COGDIS and the final four items are included only in the Cognitive Disturbances (COGDIS) high-risk profile. To fulfil COPER criteria, an individual must experience one of the first ten items at least several times in a month within the 3 months prior to assessment and the first occurrence must have been at least 12 months prior to the assessment. To fulfil COGDIS criteria, an individual must experience two of the last nine items, each at least several times in a month within the 3 months prior to assessment. Items B7, D7 and D15 can be consistently assessed in individuals aged 13 years and older.

\section{References}

1 Cipriani A, Furukawa TA, Salanti G, Chaimani A, Atkinson LZ, Ogawa Y, et al. Comparative efficacy and acceptability of 21 antidepressant drugs for the acute treatment of adults with major depressive disorder: a systematic review and network meta-analysis. Lancet 2018; 391: 1357-66.

2 Correll CU, Detraux J, De Lepeleire J, De Hert M. Effects of antipsychotics, antidepressants and mood stabilizers on risk for physical diseases in people with schizophrenia, depression and bipolar disorder. World Psychiatry 2015; 14 119-36.

3 Miura $\mathrm{T}$, Noma $\mathrm{H}$, Furukawa TA, Mitsuyasu $\mathrm{H}$, Tanaka $\mathrm{S}$, Stockton $\mathrm{S}$, et al. Comparative efficacy and tolerability of pharmacological treatments in the maintenance treatment of bipolar disorder: a systematic review and network meta-analysis. Lancet Psychiatry 2014; 1: 351-9.

4 Correll CU, Rubio JM, Kane JM. What is the risk-benefit ratio of long-term antipsychotic treatment in people with schizophrenia? World Psychiatry 2018; 17 149-60.

5 Schultze-Lutter F, Michel C, Schmidt SJ, Schimmelmann BG, Maric NP, Salokangas RKR, et al. EPA guidance on the early detection of clinical high risk states of psychoses. Eur Psychiatry 2015; 30: 405-16.

6 Zwicker A, Denovan-Wright EM, Uher R. Gene-environment interplay in the etiology of psychosis. Psychol Med 2018; 48: 1925-36.

7 Uher R, Zwicker A. Etiology in psychiatry: embracing the reality of poly-geneenvironmental causation of mental illness. World Psychiatry 2017; 16: 121-9.

8 Gandal MJ, Haney JR, Parikshak NN, Leppa V, Ramaswami G, Hartl C, et al Shared molecular neuropathology across major psychiatric disorders parallels polygenic overlap. Science 2018; 359: 693-7.

9 Cross Disorder Group of the Psychiatric Genomics Consortium. Genetic relationship between five psychiatric disorders estimated from genome-wide SNPS. Nat Genet 2013; 45: 984-94.

10 Caspi A, Houts RM, Belsky DW, Goldman-Mellor SJ, Harrington H, Israel S, et al. The $\mathrm{p}$ factor: one general psychopathology factor in the structure of psychiatric disorders? Clin Psychol Sci 2014; 2: 119-37.

11 Schultze-Lutter F, Theodoridou A. The concept of basic symptoms: its scientific and clinical relevance. World Psychiatry 2017: 16: 104-5.
12 Schultze-Lutter F, Ruhrmann S, Fusar-Poli P, Bechdolf A, Klosterkötter J. Basic symptoms and the prediction of first-episode psychosis. Curr Pharm Des 2012; 18: $351-7$

13 Schultze-Lutter F. Subjective symptoms of schizophrenia in research and the clinic: the basic symptom concept. Schizophr Bull. 2009; 35: 5-8.

14 Fux L, Walger P, Schimmelmann BG, Schultze-Lutter F. The Schizophrenia Proneness Instrument, Child and Youth version (SPI-CY): practicability and discriminative validity. Schizophr Res 2013; 146: 69-78.

15 Michel C, Ruhrmann S, Schimmelmann BG, Klosterkötter J, Schultze-Lutter F. Course of clinical high-risk states for psychosis beyond conversion. Eur Arch Psychiatry Clin Neurosci 2018; 268: 39-48.

16 Schultze-Lutter F, Michel C, Ruhrmann S, Schimmelmann BG. Prevalence and clinical relevance of interview-assessed psychosis-risk symptoms in the young adult community. Psychol Med 2018; 48: 1167-78.

17 Lo Cascio N, Saba R, Hauser M, Lammers Vernal D, Al-Jadiri A, Borenstein Y, et al. Attenuated psychotic and basic symptom characteristics in adolescents with ultra-high risk criteria for psychosis, other non-psychotic psychiatric disorders and early-onset psychosis. Eur Child Adolesc Psychiatry 2016; 25 : 1091-102.

18 Gottesman II, Laursen TM, Bertelsen A, Mortensen PB. Severe mental disorders in offspring with 2 psychiatrically ill parents. Arch Gen Psychiatry 2010; 67: 252.

19 Rasic D, Hajek T, Alda M, Uher R. Risk of mental illness in offspring of parents with schizophrenia, bipolar disorder, and major depressive disorder: a metaanalysis of family high-risk studies. Schizophr Bull 2014; 40: 28-38.

20 Forstner AJ, Hecker J, Hofmann A, Maaser A, Reinbold CS, Muhleisen TW, et al. Identification of shared risk loci and pathways for bipolar disorder and schizophrenia. PLOS One 2017; 12: e0171595

21 Selzam S, Coleman JRI, Moffitt TE, Caspi A, Plomin R. A polygenic $p$ factor for major psychiatric disorders. Transl Psychiatry 2018; 8: 205

22 Meng H, Graf Schimmelmann B, Koch E, Bailey B, Parzer P, Gunter M, et al. Basic symptoms in the general population and in psychotic and non-psychotic psychiatric adolescents. Schizophr Res 2009; 111: 32-8.

23 Klosterkötter J, Gross G, Huber G, Wieneke A, Steinmeyer EM, Schultze-Lutter F. Evaluation of the 'Bonn Scale for the Assessment of Basic Symptoms - BSABS' as an instrument for the assessment of schizophrenia proneness: a review of recent findings. Neurol Psychiatry Brain Res 1997; 5: 137-50. 
24 Uher R, Cumby J, MacKenzie LE, Morash-Conway J, Glover JM, Aylott A, et al. A familial risk enriched cohort as a platform for testing early interventions to prevent severe mental illness. BMC Psychiatry 2014; 14: 344

25 Endicott J. A diagnostic interview: the Schedule for Affective Disorders and Schizophrenia. Arch Gen Psychiatry 1978; 35: 837.

26 First MB. Structured Clinical Interview for the DSM (SCID). In The Encyclopedia of Clinical Psychology (eds RL Cautin and SO Lilienfeld): 1-6. John Wiley \& Sons, 2015.

27 Kaufman J, Birmaher B, Brent D, Rao U, Flynn C, Moreci P, et al. Schedule for Affective Disorders and Schizophrenia for School-Age Children-Present and Lifetime Version (K-SADS-PL): initial reliability and validity data. J Am Acad Child Adolesc Psychiatry 1997; 36: 980-8.

28 Schimmelmann BG, Michel C, Martz-Irngartinger A, Linder C, Schultze-Lutter F. Age matters in the prevalence and clinical significance of ultra-high-risk for psychosis symptoms and criteria in the general population: findings from the BEAR and BEARS-kid studies. World Psychiatry 2015; 14: 189-97.

29 Bates D, Mächler M, Bolker B, Walker S. Fitting linear mixed-effects models using Ime4. J Stat Softw 2015; 67: 1-48.

$30 \mathrm{R}$ Core Team. R: A Language and Environment for Statistical Computing. R Foundation, 2008

31 Schultze-Lutter F, Ruhrmann S, Michel C, Kindler J, Schimmelmann BG, Schmidt SJ. Age effects on basic symptoms in the community: a route to gain new insight into the neurodevelopment of psychosis? Eur Arch Psychiatry Clin Neurosci 2018; Oct 25 (Epub ahead of print).

32 Klosterkötter J, Hellmich M. Diagnosing schizophrenia in the initial prodromal phase. Arch Gen Psychiatry 2001; 58: 158-64.

33 van Os J, Reininghaus U. Psychosis as a transdiagnostic and extended phenotype in the general population. World Psychiatry 2016; 15: 118-24.
34 van Os J, Guloksuz S. A critique of the 'ultra-high risk' and 'transition' paradigm. World Psychiatry 2017; 16: 200-6.

35 Perlis $\mathrm{RH}$, Uher $\mathrm{R}$, Ostacher $\mathrm{M}$, Goldberg JF, Trivedi $\mathrm{MH}$, Rush J, et al. Association between bipolar spectrum features and treatment outcomes in outpatients with major depressive disorder. Arch Gen Psychiatry 2010; 68: 351.

36 Kelleher I, Keeley $\mathrm{H}$, Corcoran $\mathrm{P}$, Lynch C, Fitzpatrick N, Devlin C, et al. Clinicopathological significance of psychotic experiences in non-psychotic young people: evidence from four population-based studies. Br J Psychiatry 2012; 201: 26-32.

37 Wigman JTW, van Nierop $\mathrm{M}$, Vollebergh WAM, Lieb $\mathrm{R}$, Beesdo-Baum $\mathrm{K}$ Wittchen $\mathrm{H}$, et al. Evidence that psychotic symptoms are prevalent in disorders of anxiety and depression, impacting on illness onset, risk, and severityImplications for diagnosis and ultra-high risk research. Schizophr Bull 2012; 38: $247-57$.

38 Wigman JTW, van Os J, Abidi L, Huibers MJH, Roelofs J, Arntz A. Subclinical psychotic experiences and bipolar spectrum features in depression: association with outcome of psychotherapy. Psychol Med 2014; 44: 325-36.

39 Power RA, Kyaga $\mathrm{S}$, Uher $\mathrm{R}$, MacCabe $\mathrm{JH}$, Langstrom $\mathrm{N}$, Landen $\mathrm{M}$, et al. Fecundity of patients with schizophrenia, autism, bipolar disorder, depression, anorexia nervosa, or substance abuse vs their unaffected siblings. JAMA Psychiatry 2013; 70: 22.

40 Heckman JJ. The developmental origins of health. Health Econ 2012; 21: 24-9.

41 Bayer JK, Rapee RM, Hiscock H, Ukoumunne OC, Mihalopoulos C, Wake M. Translational research to prevent internalizing problems early in childhood. Depress Anxiety 2011; 28: 50-7.

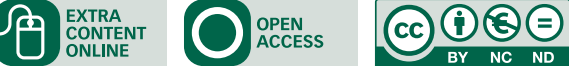

